\title{
Generalized Chebyshev-like Approximation for Low-pass Filter
}

\author{
Hisham L. Swady \\ Dept. of Electrical Engineering . \\ University of Basrah \\ Basrah, Iraq \\ Email: hisham.romi@uobasrah.edu.iq
}

\begin{abstract}
Analog filters constitute indispensible component of analog circuits and still playing an important part in interface with analog real world. realizing filters with odd order is preferred because of its time response. Therefore, this paper is conducted to introduce a new generalized Chebyshev - like approximation for analog filters. The analyses presented to realize the filters with odd order. This proposed novel approach offer good results in terms of flat delay and time domain response. Also, the achieved results are validated by comparison to normal Chebyshev filter via investigation several examples.
\end{abstract}

\section{INTRODUCTION}

The analog filter is a linear system that has an input and output signal. This main function of this system is to change the frequency response characteristics of the input signal as it moves through the filter [1]. There are many applications for analog filters, like, amplifiers, voltage controlled oscillator, converters, and sensors etc.. Also, the analog filter is the first step required to build the digital infinite impulse response (IIR) filters by using bilinear transformation or the impulse response[1-3]. The main step for designing all types of analog filters should start with present a normalized platform. Then, the designer should be able to realize the achieved platform with the required scale. The standard normalization located within the range of $0 \sim 1$ and stopband located in $\left[w_{s},+\infty\right][4,5]$.

A considered research papers have been conducted to address several efficient optimization techniques [6 - 8]. The exits methods based on utilizing analog platform to be used in realizing the digital filters, or employing some digital filters platform for realizing. So, to achieve improvement for both types of filters this paper has been conducted to present the required steps for designing analog filters which can be based in building its counterpart of filters- digital filters. This work presents a novel general approach for analog filter designing for Chebyshev-like approximation with odd order. Chebyshev-like filters differs from classical Chebyshev ones in the ripple factor, $\varepsilon$, which is not equal amplitudes with classical one.

\section{THE PROPOSED APPROXIMATION}

Consider the following general magnitude squared function of a low- pass filter of order $\mathrm{N}=2 n+1[9]$ :

$$
|F(j w)|^{2}=\frac{1}{1+\varepsilon^{2} P\left(w^{2}\right)}
$$

$$
\begin{aligned}
& \text { Where } \\
& \varepsilon^{2}=\delta p^{-2}-1 ;
\end{aligned}
$$

$\delta \mathrm{p}$ is the minimum value of $|F(j w)|$ in the passband $0 \leq w \leq 1$. The classical approach to obtain a low-pass characteristic is based on searching for a function similar to Eq. 1 and satisfy the following conditions [9]:

$$
\begin{gathered}
\varepsilon^{2} P\left(w^{2}\right) \ll 1 \quad 0<<w<<w_{p} \\
\varepsilon^{2} P\left(w^{2}\right) \gg 1 \quad w>w_{s}
\end{gathered}
$$

Our approach depends on choosing $\delta p \leq|F(j w)| \leq 1$ in the passband. After several manipulations the formula is obtained:

$$
\max \left[P\left(w^{2}\right)\right] \leq 1
$$

For a special case with choosing $p\left(w^{2}\right)=C_{N}^{2}(w)$ equation 1 becomes identical to the magnitude squared function of the Chebyshev filter[10] as: 


$$
|F(j w)|^{2}=\frac{1}{1+\varepsilon^{2} C_{N}^{2}(w)}
$$

The value of $n$ is determined from the stopband requirement that satisfy:

$|F(j w)| \leq \delta_{s}, w \geq w_{s}$

Where $w_{s}$ and $\delta_{s}$ denote the prescribed stopband edge frequency and the stopband tolerance, respectively.

Eq.5 can be transformed into the following inequality by an analytical manipulation which results:

$\mathrm{P}\left(\mathrm{w}^{2}\right) \geq \frac{\delta_{s}^{-2}-1}{\delta_{p}^{-2}-1}$

Numerical techniques should be used to evaluate equations 3 and 6 to calculate the filter parameters after proper choice for $p\left(w^{2}\right)$ is achieved.

\section{VALIDATE THE PROPOSED SCHEME}

To validate the proposed approach $p\left(w^{2}\right)$ should be pointed as a first step. Rf. [11] offered the following rational function:

$P\left(w^{2}\right)=w^{2} \prod_{i=1}^{n}\left[\frac{w^{2}-w i^{2}}{1-w_{i}^{2}}\right]^{2}$

This equation will be based in the prototype. Thus, the design procedure as follows:

Given the design specifications: maximally allowable passband ripple and maximally allowable stopband ripple. The Chebyshev like filter of odd filer $n$ can be found as:

- Step 1. Maximize Eq. 3 for calculating $w_{i}$.

- Step 2. Substituting the results into Eq. 6 for getting $w_{s}$.

- Step 3. The pole locations of the new filter will be obtained by using this equation[11]:

$$
s \prod_{i=1}^{n}\left(s^{2}-w_{i}{ }^{2}\right)= \pm \prod_{i=1}^{n}\left(1-w_{i}{ }^{2}\right) / \varepsilon
$$

\section{DESIGN EXAMPLES}

Let us consider the following design examples for illustrating the proposed scheme.

Example 1:

For $n=1$, and $\mathrm{N}=3$, with Eq. 3, the maximum value of $\max \left[w^{2}\left(w^{2}-w_{1}^{2}\right)^{2}\right]$

gives $w=0.866 w_{1}$, then the inequality of Equ. 3 becomes:

$2\left[w_{1}-(0.866)\right]\left(w_{1}+1.7\right)^{2} \leq 0$

Thus, $w_{1} \leq 0.866$ should satisfy inequality in eq. 10 .

Thus, Eq.(6) be as :

$w_{s}^{3}-w_{1}^{2} w_{s}-k\left(1-w_{1}^{2}\right) \geq 0$

Then the optimum solution of this condition is $w_{1}=0.8$ and $w_{s}=3.2$ and the pole locations of the new filter are -0.665 and $-0.333 \mp \mathrm{j} 0.986$ for $\delta \mathrm{p}=0.894$.

The magnitude response of both of the Chebyshev and Chebyshev-like are shown in Fig.1. Fig.1 shows passband performance of the proposed design and the classical Chebyshev filters under the same specifications $(\delta p=0.894)$. It's clear that the new proposed approximation has non- equal ripple factor in the passband and more flatness than Chebyshev filter as shown in Fig.2. Furthermore, the improvement in the time domain response can be observed.

Similar procedure is applied also, for $\delta p=0.891$ and the results are shown in Fig.3. The passband ripple factor and is illustrated for range of frequencies, the ripple also is not equal and there is an improvement in flatness of the response as shown in Fig.4.

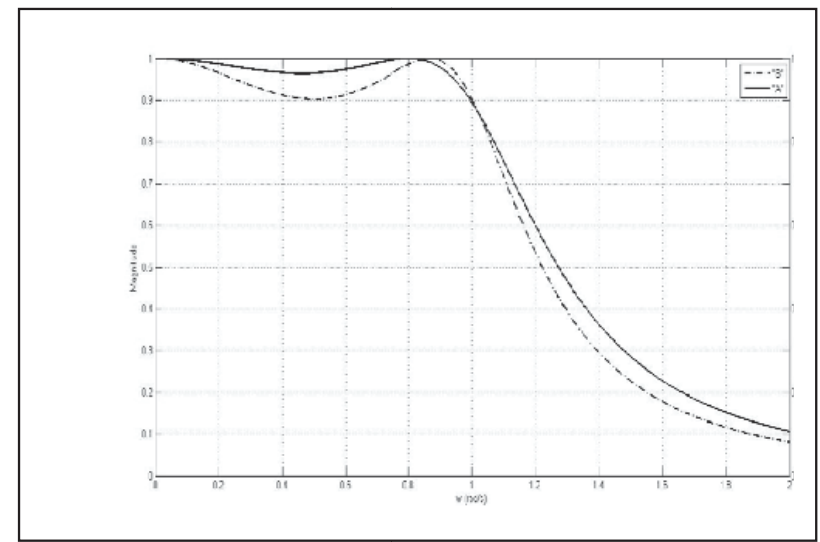

Fig.1: Passband performance of the proposed design filter ("A") and the Chebyshev filters ("B") for $\delta_{\mathrm{p}}=0.894,3$ rd order. 


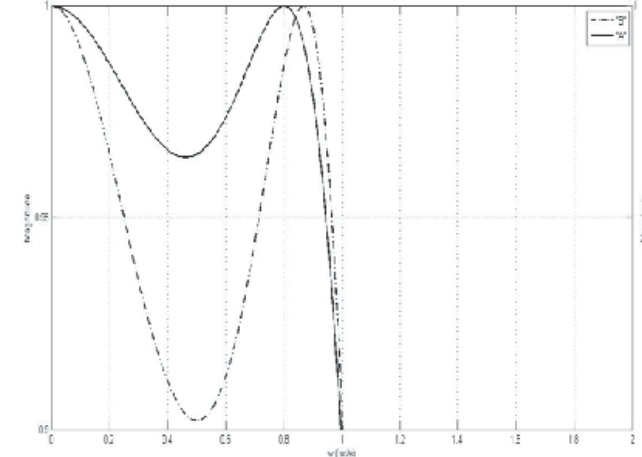

Fig. (2) Passband details comparison between proposed design filter ("A")and the Chebyshev filters("B") for $\delta_{\mathrm{p}}=0.894,3$ rd order.

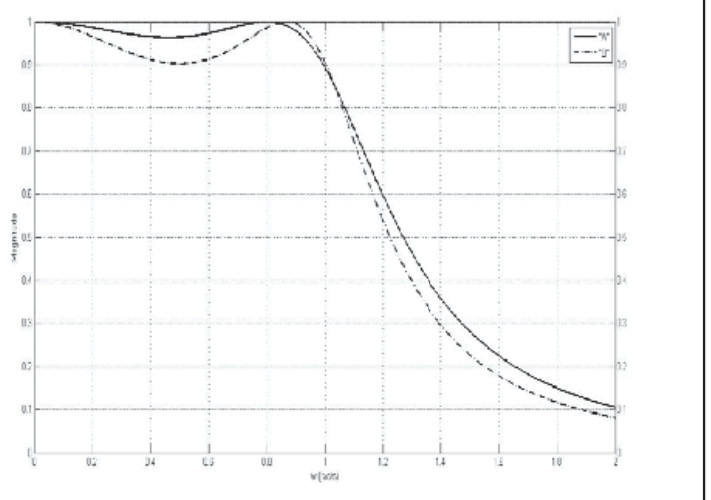

Fig. (3) Passband performance of the proposed filter ("A") and the classical Chebyshev filters ("B") $\delta_{\mathrm{p}}=0.891$, 3rd order.

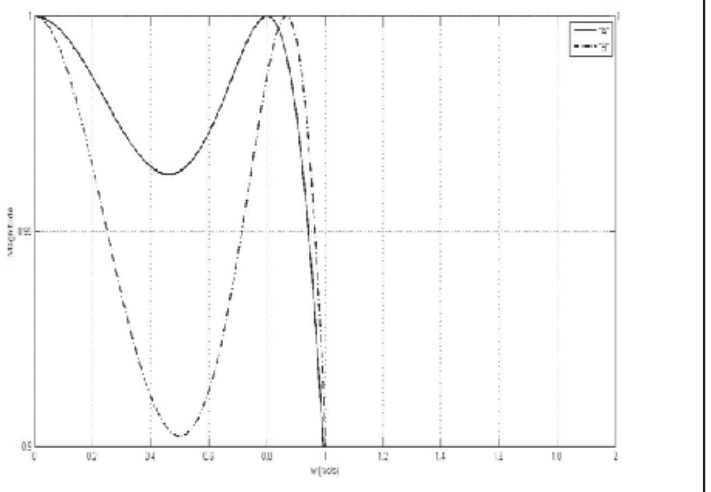

Fig. (4) Passband details comparison between the proposed filter design ("A")and the Chebyshev filters("B") for $\delta_{\mathrm{p}}=0.891,3 \mathrm{rd}$. order.
Example 2:

For $n=2, \mathrm{~N}=5$ used with eq.3, the following equation should be maximize: $\max \left[w^{2}\left(w^{2}-w_{1}{ }^{2}\right)^{2}\left(w^{2}-w_{2}{ }^{2}\right)^{2}\right]$ ..(12)

With utilizing numerical techniques to evaluate $\mathrm{w}_{\mathrm{i}}$ 's. $w_{1}=0.5$ and $w_{2}=0.9$ are obtained.

Then, the pole locations of the new filter are:-0.5143, $-0.1449 \pm \mathrm{j} 1.0056$ and $-0.407 \pm \mathrm{j} 0.6076$ for $\delta \mathrm{p}=0.894$.

The magnitude response of the two filters are shown in Fig. 5.

The higher order filter design with the new filter offer lower ripple magnitude than that with classical Chebyshev filter. But this ripple is increased as $\mathrm{w}$ increases from 0 to 1 for the high order filters. This can be shown clearly in Figs. 5 and 6. Fifth order is also considered for $\delta p=0.891$ and the obtained results are shown in Figs. 7 and 8. It show that ripple factor gained clear improvement compare to the classical one.

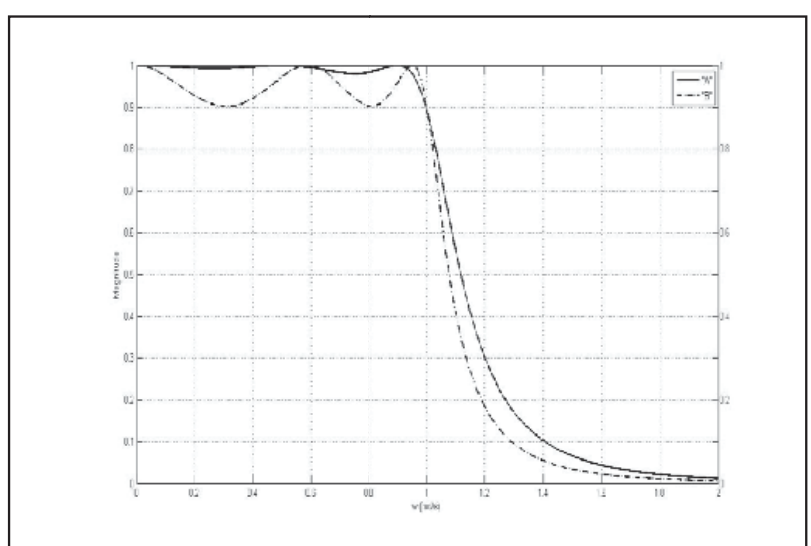

Fig.5: Passband performance of the proposed design filter ("A") and the Chebyshev filters("B"), for $\delta_{\mathrm{p}}=0.894$, 5 th order. 


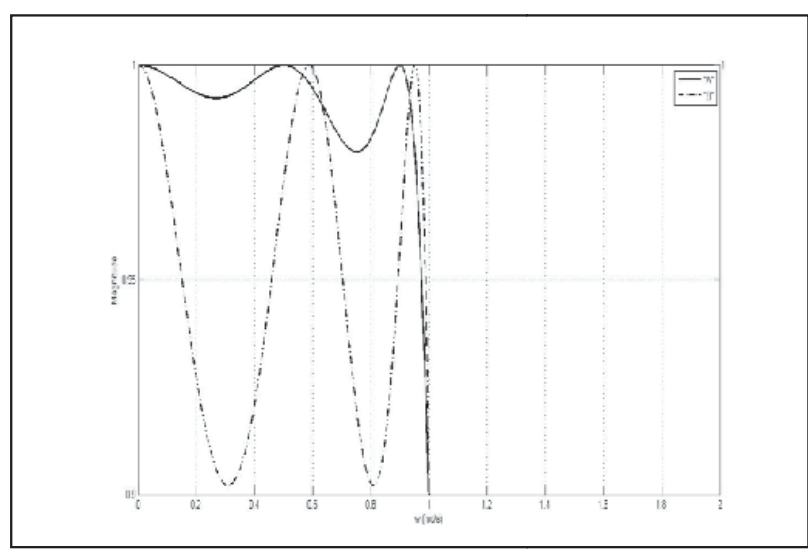

Fig.6 : Passband details comparison between the proposed design filter ("A")and the Chebyshev filters("B") for $\delta_{\mathrm{p}}=0.894,5$ th order.

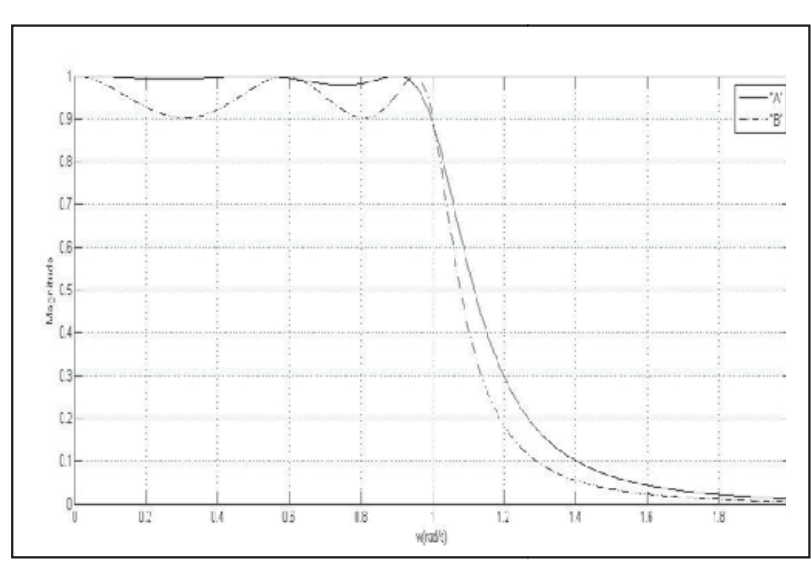

Fig. 7: Passband performance of the proposed design filter ("A") and the Chebyshev filters("B") for $\delta_{\mathrm{p}}=0.891,5$ th order.

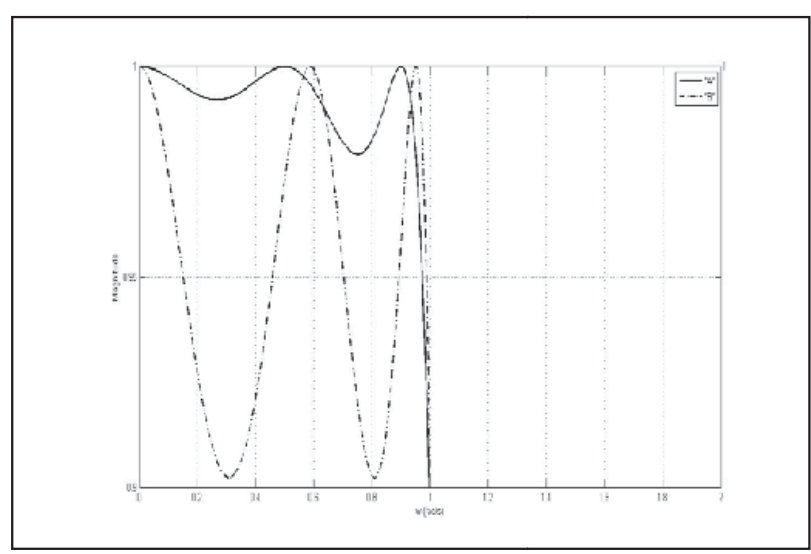

Fig. 8: Passband details comparison between the proposed design filter ("A") and the Chebyshev filters("B") for $\delta_{\mathrm{p}}=0.891,5$ th order.

\section{CONCLUSION}

In this paper, we have addressed a generalized Chebyshev-like approximation for low-pass filter design. The approach for one approximation method is compared with those of the classical Chebyshev filter. The obtained results show that the proposed designed filter gives lower $\mathrm{Q}$ values of complex poles with a considerable value of flatness of the filter passband response.

\section{REFERENCES}

[1] H. K. Kim, "The effects of lower Q values on the filters having equal ripples in the passband", IEEE Trans. Circuits Syst., Vol. 39, pp. 305-309, 1992.

[2] F.F. Kuo, Network Analysis and Synthesis. New York, Wiley, 1977.

[3] U.Tietzem C.Shank, E.Gamm, Handbook of Electronic Circuits. Berlin: Springer, 2008.

[4] A. Antniou, Digital signal processing. New York: McGrawHill, 2006.

[5] S. Winder, Analog and Digital Filter Design, 2nd, Newnes, 2002.

[6] N. T. Hoang, H.D. Tuan, T. Q. Nguyen, and H. G. Hoang," Optimized Analog Filter Designs With Flat responses by Semidefinite Programming", IEEE Trans. Signal Processing, Vol. 57, No. 3, March 2009.

[7] T. Iwasaki and S. Hara, "Generalized KYP lemma: Unified frequency domain inequalities with design applications," IEEE Trans. Autom. Control, vol. 50, no. 1, pp. 41-59, Jan. 2005.

[8] N. T. Hoang, H.D. Tuan, T. Q. Nguyen, and H. G. Hoang," Optimized Analog Flat Filter Design", IEEE Trans. Signal Processing, Vol. 57, No. 3, March 2009.

[9] K.Su, Analog Filters, 2nd. Kluwer Academic publishers, 2002.

[10] M. Weeks, Digital Signal Processing using MATLAB and Wavelets. Infinity Science press,2007.

[11] S.C. Dutta Roy, "A New Chebyshev-like Low-pass Filter Approximation" , Springer Science+ Business Media, LLC2010, Circuit Syst. Signal process Vol. 29: pp 629-636. 